\title{
SECRETORY IMMUNOGLOBULIN A (SIGA) AND DENTAL CARIES OF CHILDREN WITH DIFFERENT DISEASES AND CONDITIONS INFLUENCING ORAL MEDIUM
}

\author{
Rashkova M*, M. Baleva**, M. Peneva*, N. Toneva*, G. Jegova* \\ *Department of Paediatric Dentistry, Faculty of Dental Medicine, MU-Sofia \\ ** Department of allergology, Laboratory of clinical immunology, MU -Sofia
}

\section{SUMMARY}

SIgA is the main type immunoglobulin in the mixed saliva and is considered as the main secretion factor of the adaptive immunity in the mouth. The purpose of the study is an evaluation of the SIgA quantities and the connection of those antibodies with dental caries of children with different diseases and conditions influencing the oral medium. The study was performed with 116 children with diabetes, asthma, orthodontic problems (removable braces) and healthy controls. The following methods were used: (1) ELISA with "Salivary secretory IgA KIT of Salimetrics LLC USA; was used for quantitative determination of IgA$\mathrm{S}$ in the saliva ;(2) Evaluation of the liquid oral medium (test "Saliva check-GC") and dental status (DMF-T) of the children studied.

The results obtained came to show that $2 / 3$ of the healthy children and the children with diabetes have low values of $\mathrm{c}(<100 \mu \mathrm{g} / \mathrm{ml})$. In the group of healthy children there are no high values of SIgA; Middle values of SIgA $(100-300 \mu \mathrm{g} / \mathrm{ml})$ are most frequently met in children with asthmatic disease; Oone half of the children with orthodontic apparatuses have high values of SIgA $(>300$ $\mu \mathrm{g} / \mathrm{ml}$ ); There is no dependence between secretory immunity and dental caries in children.

Key words: secretory IgA-antibodies, oral homeostasis, dental biofilm, diabetes, asthma, orthodontic treatment, dental caries, DMF.

\section{INTRODUCTION}

Secretory immunoglobulin A ( $\operatorname{SIgA})$ is the dominant immunoglobulin in external secretions and also in mixed saliva. It is often characterized as a component of the immune system "first-line of defense" against pathogenic microorganisms. Restricting microbes'adhesion, these antibodies react to dental biofilm formation and thus interfere into the defense of plaque-oral pathology (caries and periodontal diseases $)(1,2,6,8)$.

SIgA is an essential biomarker for the local defense of the mouth. Their secretion depends on the general health of the organism and its immunity. On the other hand, the rich oral antigen potential is also a stimulus for SIgA antibodies $(3,4,5,9)$.

The role of SIgA for the development of dental caries is determined by the ability of such antibodies to impede plaque microorganisms' colonization on the enamel surface by selective connection. Thus they oppose all other mechanisms of microbe adhesion and interfere during formation of plaque biofilm(9). An interesting question is why residual microflora persists in oral cavity despite the presence of a high SigA level in the saliva? It is considered that the stability of the flora is a result of its decreased activity to provoke immune reactions. It is assumed that the greater part of the oral microorganisms are not immunogenic for the organism since they live in symbiosis with the body following the long period of evolutionary adaptation $(10,13)$.

It is the aim of the investigation to evaluate the quantities of SIgA in children with different diseases and conditions influencing the oral environment as well as the connection of these antibodies with dental caries.

For the purpose we set the following tasks:

1. Study of the average values of SIgA in the saliva of children with different diseases and conditions influencing oral medium;

2. Study of the dependence between dental caries and SIgA in the children under investigation.

\section{MATERIALAND METHODS}

The study was performed on 116 children: (1)30 diabetics children (av. age 12,2 3,53 years old);(2) 25 children suffering asthma (av. age 8,84 $\pm 3,02$ years old);(34)

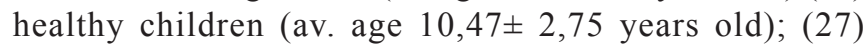
undergoing orthodontic treatment (av. age 11,04 $\pm 1,26$ years old).

The selected groups of children are suitable for this study because in the three of them we can expect a change in the oral immunity. In diabetic children this change is in relation to the main disease; in asthmatic children- allergic terrain and local influence of the inhalatory corticosteroids; the local irritating effect of the plastic in the orthodontic 
appliances may modify the oral medium(12).

\section{Clinical examination and evaluation of dental}

caries. All children were subjected to:

- evaluation of the oral medium by tools of caries risk assessment(11);

- registration of the dental caries $-\mathrm{DMF}(\mathrm{T}+\mathrm{t})$;

- saliva sample for SIgA study was taken.

2. Method of saliva collection. Saliva was collected in the morning, on empty stomach, after stimulation for 2 min the salivation by means of chewing standard neutral chewing gum (from the test "Saliva Chek" GC. Stimulated saliva was collected in a plastic container of $5 \mathrm{ml}$ and then by a dropper a certain amount was taken and frozen in a refrigerator $\left(-10^{\circ} \mathrm{C}\right)$.

3. ELISA method for study of SIgA in the saliva(14). For the quantitative determination of the SIgA in the saliva we employed the ELISA method with „Salivary secretory IgA KIT" of SalimetricsLLC - USA. The method is indirect, a constant quantity of goat anti-human SIgA conugated with horseradish peroxidase(HRP) being used. The antibody-conjugate is added in test-tubes with specific dilutations of standards or samples from the saliva studied. From each sample sprinkling is made in separate small holes with human SIgA grouped on the same plaque. The quantities of free antiobodies are in reverse proportion to the quantity of SIgA. An enzyme reaction is evoked, which causes blue colouring that changes into yellow after the end of the reaction. By means of standard registration as per ELISA the optical thickness is measured with a length of the wave $450 \mathrm{~nm}$ and $650 \mathrm{~nm}$, the average value being taken into account. After certain calculations (described by the firm producer of the test) a standard curve is built from the values of the six controls and the standards. By means of the equation composed on the basis of the curve the concentrations of SIgA are calculated in $\mu \mathrm{g} / \mathrm{ml}$.

Fig. 1. Standard curve obtained from the study

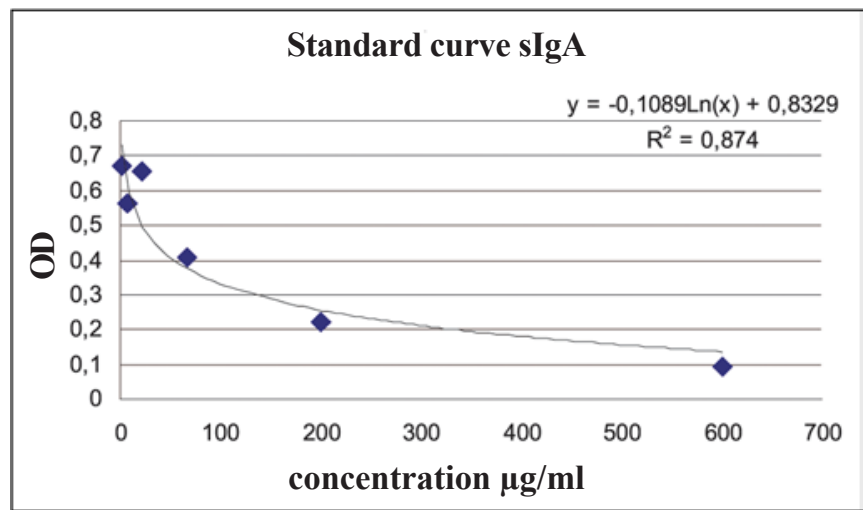

The statistical processing was done with the help of the computer program SPSS 16.

\section{RESULTS}

1. Comparative study of SIgA in the saliva of children studied. The average values of SIgA in the saliva of the healthy children (third group) are 121,34 $\pm 15,027 \mu \mathrm{g} /$ $\mathrm{ml}$. For children with diabetes (first group) the average quantities of SIgA in the saliva are $133,95 \pm 160,504 \mu \mathrm{g} / \mathrm{ml}$, close to those of the control group. Due to the initial phase of diabetes development, the system endocrine disease seems not to influence the local secretory oral immunity. Children with asthma exhibit statistically verifiable higher quantities of IgA-S $(196,456 \pm 145,308 \mu \mathrm{g} /$ $\mathrm{ml})$, compared to the healthy children $(\mathrm{P}<0,05)$. The average values of SIgA in children with orthodontic apparatuses are even higher $(208,874 \pm 125,953 \mu \mathrm{g} / \mathrm{ml})$. The results can be explained by the stimulating action of the plastic apparatus, alien to the oral environment or by the conditions created in the mouth that lead to more difficult maintenance of the oral hygiene, thus enforcing the bacterial antigenic stimulus.

We distributed the examined children in the following groups:

(1) low SIgA(up to $100 \mu \mathrm{g} / \mathrm{ml}$ );

(2) medium SIgA(100 - $300 \mu \mathrm{g} / \mathrm{ml})$;

(3) high SIgA (>300 $\mu \mathrm{g} / \mathrm{ml})$ values of SIgA.

The distribution of the children examined into the groups thus formed is shown in the following table.

Fig. 2. Distribution of the children examined according to the quantity of SIgA in the saliva

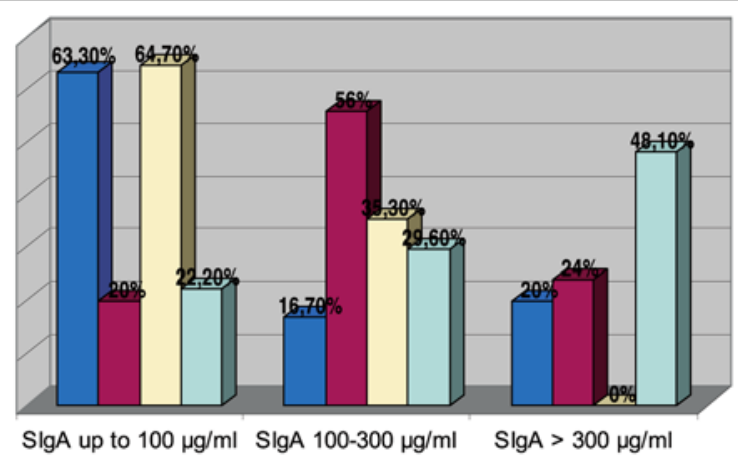

$\square$ with diabetes $\square$ with asthma $\square$ healthy $\square$ with orthod. Apparatures

The results obtained show that in the group of healthy children are absent any high values of SIgA ( $>300$ $\mu \mathrm{g} / \mathrm{ml})$. Two thirds $(64,7 \%)$ of these children are with low and $1 / 3(35,3 \%$ ) with medium values of SIgA (up to $300 \mu \mathrm{g} /$ $\mathrm{ml}$ ). In the two group with proven above the average values of SIgA (children with asthma and with orthodontic apparatuses) a very interesting distribution of data is observed - half of the children with asthma $(56 \%)$ belong to the category with medium values, accordingly, half of the children with orthodontic apparatuses $(48,1 \%)$ belong 
to the category with high values. The distribution of the low values in the children with diabetes is similar to that of healthy children, but unlike the healthy children in $1 / 5$ of the children with diabetes $(20 \%)$ there also very high values (> $300 \mu \mathrm{g} / \mathrm{ml})$.

\section{Study of the dependence between dental caries} and SIgA. In order to investigate the dependence of SIgA and the dental caries in clinical conditions, we followed DMF $(\mathrm{T}+\mathrm{t})$ by grouping the children studies in two ways:

- In the mentioned above three groups according to SIgA:

(1)low SIgA(up to $100 \mu \mathrm{g} / \mathrm{ml}$ );

(2)medium SIgA(100 -300 $\mu \mathrm{g} / \mathrm{ml})$;

(3)high $\operatorname{SIgA}(>300 \mu \mathrm{g} / \mathrm{ml})$ values of SIgA.

- In two groups according to their cariosity:

- DMF $(\mathrm{T}+\mathrm{t})$ up to 6 teeth;

- DMF $(\mathrm{T}+\mathrm{t})$ above 6 teeth.

2.1. $D M F(T+t)$ in children grouped according to the quantities of SIgA. To eliminate the influence of the age factor, in addition to the curiosity, we compared the average age of the children in the groups formed. The results are shown in the next table.

Table 2. Evaluation of the average age of the children divided according to the quantities of SIgA

\begin{tabular}{|l|l|l|l|l|}
\hline \multirow{2}{*}{} & \multirow{2}{*}{$\mathrm{n}$} & \multicolumn{3}{|c|}{ Age - years } \\
\cline { 3 - 5 } & $\mathrm{X}$ & $\pm \mathrm{SD}$ & $\Delta$ \\
\hline 1st group & 52 & 10,84 & 3,07 & 0,421 \\
\hline 2nd group & 39 & 10,56 & 3,34 & 0,535 \\
\hline 3rd group & 25 & 10,65 & 2,49 & 0,499 \\
\hline T P & $\mathrm{n}$ & \multicolumn{4}{|c|}{$\mathrm{P}>0,05$} \\
\hline
\end{tabular}

It should be noted that though the average age of the children does not differ in the three groups $(\mathrm{P}>0,05)$.

Dental caries(DMF) is approximately equal for children with very low and very high values of SIgA. For the group with children with average values, the DMFindex is lower, and the differences are not statistically verifiable $(\mathrm{P}>0,05)$.
Fig. 3. $\operatorname{DMF}(\mathrm{T}+\mathrm{t})$ of children divided to the quantities of SIgA

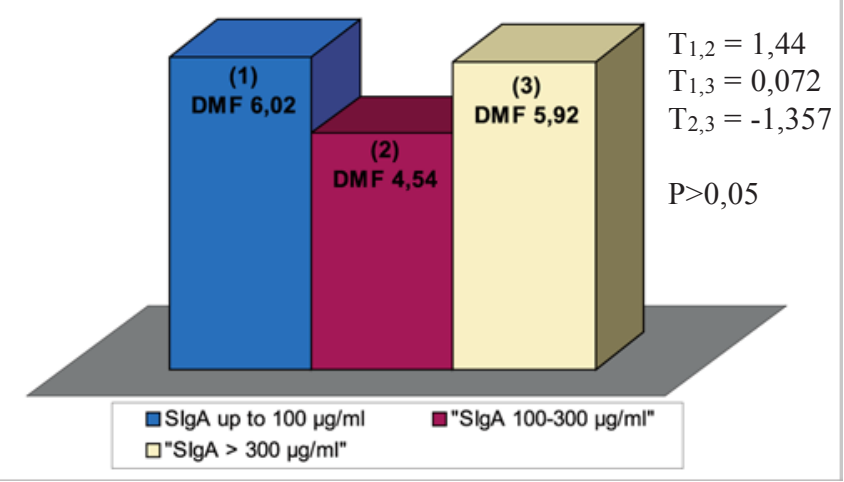

These results show a lack of dependence between the secretory immunity and dental caries. This conclusion is confirmed also when comparing SIgA of the children grouped according to $\mathrm{DMF}(\mathrm{T}+\mathrm{t})$.

2.2. Comparing SIgA of the children grouped according to $\mathbf{D M F}(\mathbf{T}+\mathbf{t})$. The results are shown in Table 3 .

Table 3. SIgA of children studied according to $\operatorname{DMF}(\mathrm{T}+\mathrm{t})$

\begin{tabular}{|l|l|l|l|c|}
\hline \multirow{2}{*}{$\begin{array}{l}\text { Cariosity } \\
\text { DMF-T }\end{array}$} & \multirow{2}{*}{$\begin{array}{l}\text { Number of } \\
\text { children (n) }\end{array}$} & \multicolumn{3}{|c|}{ IgA-S $\mu \mathrm{g} / \mathrm{ml}$} \\
\cline { 3 - 5 } & & $\mathrm{X}$ & $\pm \mathrm{SD}$ & $\Delta$ \\
\hline $\begin{array}{l}\text { DMF(T+t) } \\
\text { up to 6 }\end{array}$ & 82 & 150,02 & 132,33 & 14,61 \\
\hline $\begin{array}{l}\text { DMF }(\mathrm{T}+\mathrm{t}) \\
\text { above 6 }\end{array}$ & 33 & 160,94 & 143,36 & 24,95 \\
\hline T P & $\mathrm{T}=-0,391$ & $\mathrm{P}=0,697$ & $(\mathrm{P}>0,05)$ \\
\hline
\end{tabular}

There are no proven differences between SIgA of children with lower and those with higher cariosity.

\subsection{Correlation dependence between $\operatorname{DMF}(T+t)$ and}

SIgA. We searched for the presence of correlation dependence between DMF $(T+t)$ and SIgA. The coefficient of Pearson showed very low values which confirm the lack of dependence between caries and SIgA.

Table 4. Correlation between DMF and SIgA

\begin{tabular}{|l|l|}
\hline Pearson correlation & SIgA \\
\hline $\operatorname{DMF}(\mathrm{T}+\mathrm{t})$ & 0,032 \\
\hline
\end{tabular}




\section{DISCUSSION}

For the first time in our country a study has been performed on the local secretory immunity as a part of the complex evaluation of the oral risk environment in children with different diseases. The differences in the average values shows that the secretory immunity is stimulated more than the local immunogenic factors and the immunopathological processes (asthma) and is not influenced by system diseases like diabetes. Plastic orthodontic apparatuses as local antigenic irritators provide greatest stimulus on oral secretory immunity.

No dependence was found between the secretory immunity and dental caries in children. The role of SIgA in the development of dental caries is determined by the ability of these antibodies to selectively connect with plaque microorganisms and thus impede their colonization on the enamel surface. In this way they counteract to all other mechanisms of microbe adhesion and impede the formation of plaque biofilm. Secretory $\operatorname{IgA}$ is connected mostly with
S.mutans and antigens of its enzymes and metabolic products.

In real oral environment however this at first glance considerable preventive action of SIgA with respect to dental caries is not so efficient due to the constant washing off action of saliva and the impossibility to maintain sufficient concentration of SIgA on the enamel surface.

\section{CONCLUSIONS:}

1. $2 / 3$ of the healthy children and the children with diabetes have low values of $\mathrm{c}(<100 \mu \mathrm{g} / \mathrm{ml})$. In the group of healthy children there are no high values of SIgA;

2. Middle values of SIgA (100 $-300 \mu \mathrm{g} / \mathrm{ml})$ are most frequently met in children with asthmatic disease;

3 . One half of the children with orthodontic apparatuses have high values of $\operatorname{SIgA}(>300 \mu \mathrm{g} / \mathrm{ml})$;

4. There is no dependence between secretory immunity and dental caries in children.

\section{REFERENCES:}

1. Biesbrock, A. R., M. S. Reddy, and M. J. Levine. Interaction of a salivary mucin-secretory immunoglobulin A complex with mucosal pathogens. Infect. Immun. 1991, 59,(10), 3492-3497.

2. Bokor-Bratiж M. Clinical significance of analysis of immunoglobulin A levels in saliva, Med Pregl.53,(3-4),164-8.

3. Brandtzaeg P.Do salivary antibodies reliably reflect both mucosal and systemic immunity? Ann N Y Acad Sci. 2007, 1098, (3), 288-311.

4. Brandtzaeg, P. Molecular and cellular aspects of the secretory immunoglobulin system. Acta Pathol. Microbiol. Immunol. Scand. 1995.103,(1),1-19.

5. Childers, N. K., M. G. Bruce, and J. R. McGhee. Molecular mechanisms of immunoglobulin A defense. Annu. Rev. Microbiol. 1989, 43, (5),503-536.
6. Dodds MW, Jonson DA, Yeh CK. Health benefits of saliva: a review. J Dent 2005; 33(3)223-33.

7. Herenia P. Lawrence, DDS, MSc, PhD - Salivary Markers of Systemic Disease: Noninvasive Diagnosis of Disease and Monitoring of General Health. J Can Dent Assoc 2002; 68(3):170-4.

8. Malamud Daniel. Salivary diagnostics The future is now. J Am Dent Assoc . 2006, 137, (3), 284-286.

9. Marcotte H., Marc C. Lavoie - Oral Microbial Ecology and the Role of Salivary Immunoglobulin A. Microbiol Mol Biol Rev,1998, 62,(1), 71-109.

10. Nauntofte B, Tenevuo J. O., Lagerlцf F. Secretion and composition of saliva. In: Fejerskov O. and Kidd E., eds. Dental Caries. The disease and its clinical management. Oxford. Blackwell
Munksgard; 2003. p. 7-29.

11. Rashkova M., M. Peneva, L. Doychinova, Study of the risk factors for the development of dental caries and creation of a system for assessment the risk of caries in children in Bulgaria OHDMBSC, 2008, 7,(2) - June,3-11.

12. Rashkova M., M. Peneva, M. Baleva, N. Toneva. M. Belcheva, Study if oral biomarkers and candida in the oral ecosystem in childhood. Project No. 53/ 2007 supported by GRANT - Medical University, Sofia, 96p.

13. Tenovuo J. O. Salivary parameters of relevance for assessing caries activity in individuals and populations. Comm Dent Oral Epidemiol 1997; 25(8)82-6.

14. www.Salimetrics.com - Salivary secretory IgA indirect enzyme immunoassay Kit Catalog ) 1-1602.

\footnotetext{
Address for correspondence:

Maya Rashkova, Associate professor, DDS, Ph.D

Department of Pediatric Dentistry,

Faculty of Dental medicine, Medical University-Sofia,

Address: 1, G. Sofiyski Str., Sofia 1000, Bulgaria.

E-mail: mayarashkova@mail.bg

Mobile: +359 888215033
} 